\title{
Prognostic Value of Low Diastolic 24-H Blood Pressure in Type 2 Diabetic Patients in a Follow-Up of 4.7 Years
}

\author{
Tiago G. Teixeiraa, ${ }^{\text {a }}$, Jose M. Bastos ${ }^{\mathrm{a}, \mathrm{b}}$, Jose A. Santos ${ }^{\mathrm{a}}$, Jorge Polonia ${ }^{\mathrm{c}}$
}

\begin{abstract}
Background: The prognostic implications of the 24 hours ambulatory blood pressure monitoring (AMBP) have been progressively established but some issues remain controversial about the prognostic value of circadian blood pressure (BP) profile in some highrisk populations, namely the diabetics.
\end{abstract}

Methods: We followed, for $4.7 \pm 3.5$ years, 262 type 2 diabetic patients without previous cardiovascular events after AMBPs, on the occurrence of a combination of coronary events and stroke.

Results: There were 36 events (18 coronary and 18 strokes), 7 of them fatal. Half the population showed a non-dipping pattern (nondippers plus reverse dippers) and the later conveyed a lower survival rate free from strokes $(\log$ rank 6.99; $\mathrm{P}<0.01)$ as related to the dipper patients, in the Kaplan-Meier curves. There was a clear $\mathrm{J}$ shaped curve for the distribution of the 24-hour diastolic blood pressure (24H DBP) for global and coronary events, that persisted even after the exclusion of patients with isolated systolic hypertension. Furthermore, in the subgroup with higher $(>75 \mathrm{mmHg}) 24 \mathrm{H}$ $\mathrm{DBP}$, but not in the group with $24 \mathrm{H}$ DBP below $75 \mathrm{mmHg}$, the $24 \mathrm{H}$ systolic BP value was an independent predictor of global events in the multivariate analysis (HR 0.03; 95\%CI $0.09-0.57$; $<<0.002$ ), showing decreased event-free survival in the Kaplan-Meier curves (log rank 9.15; P $<0.003)$.

Conclusions: In our population of diabetic type 2 patients $24 \mathrm{H}$ DBP had an impact on prognosis on its own, particularly for its lower values. Thus low values of $24 \mathrm{H}$ DBP are associated with high cardiovascular risk.

Manuscript accepted for publication February 18, 2013

${ }^{\mathrm{a} C e n t r o ~ H o s p i t a l a r ~ B a i x o ~ V o u g a, ~ P o r t u g a l ~}$

${ }^{\mathrm{b}}$ Universidade de Aveiro, Portugal

${ }^{\mathrm{c}}$ Universidade de Medicina do Porto, Portugal

${ }^{\mathrm{d} C o r r e s p o n d i n g ~ a u t h o r: ~ T i a g o ~ G . ~ T e i x e i r a, ~ R u a ~ d o ~ L i l a, ~ n ~ 5 ; ~} 1$ N; bloco

B - 3810-427, Aveiro, Portugal. Email: tiagoteixeiranoc@gmail.com

doi: http://dx.doi.org/10.4021/jem155e
Keywords: AMBP; Low diastolic 24-hour BP; Type 2 diabetics

\section{Introduction}

Prevalence of type 2 diabetes is continually increasing, due to aging, population growth, urbanization, and increasing prevalence of other diseases such as obesity [1]. Diabetes is also a matter of public health concern since it is associated with a high risk for all cause mortality, mainly due to cardiovascular disease [2]. The type 2 diabetes status contributes by itself to excessive cardiovascular risk [3], but the individual risk is conveyed by the interaction of other risk factors that may be present [4], hypertension being one of the key factors for the development of diabetic complications [5].

Meanwhile, most of the studies evaluating the impact of hypertension as a cardiovascular risk factor were based on a few blood pressure (BP) measurements, in the clinical setting, despite growing recent evidence supporting ambulatory monitoring of 24 hours blood pressure (AMBP) to be more useful for the prognostic assessment of the cardiovascular risk $[6,7]$. Also, AMBP has been particularly useful for the diagnostic of white coat hypertension and for the characterization of abnormal circadian BP rhythms (dipping versus non-dipping patterns) [8]. However there are some unsolved issues related to AMBP reproducibility and to the prognostic impact of each of the AMBP parameters/indexes namely in the diabetic population. Nighttime BP has been shown to be superior to daytime BP values in assessing cardiovascular risk in the general population $[9,10]$. In a small study it was shown that the nocturnal rise of BP may lead to microvascular complications in diabetics [11], whereas diabetes is commonly associated with a non-dipper pattern. Moreover, it is still a matter of debate the potential cardiovascular dangers of lowering BP too aggressively [12] (particularly of diastolic BP), its negative effect on cardiac events, and either in patients with [13] or without [14] proved ischemia and what is the threshold below which the cardiovascular risk increases.

Our objective was, therefore, to evaluate the prognostic implications of AMBP in a type 2 diabetic population for 


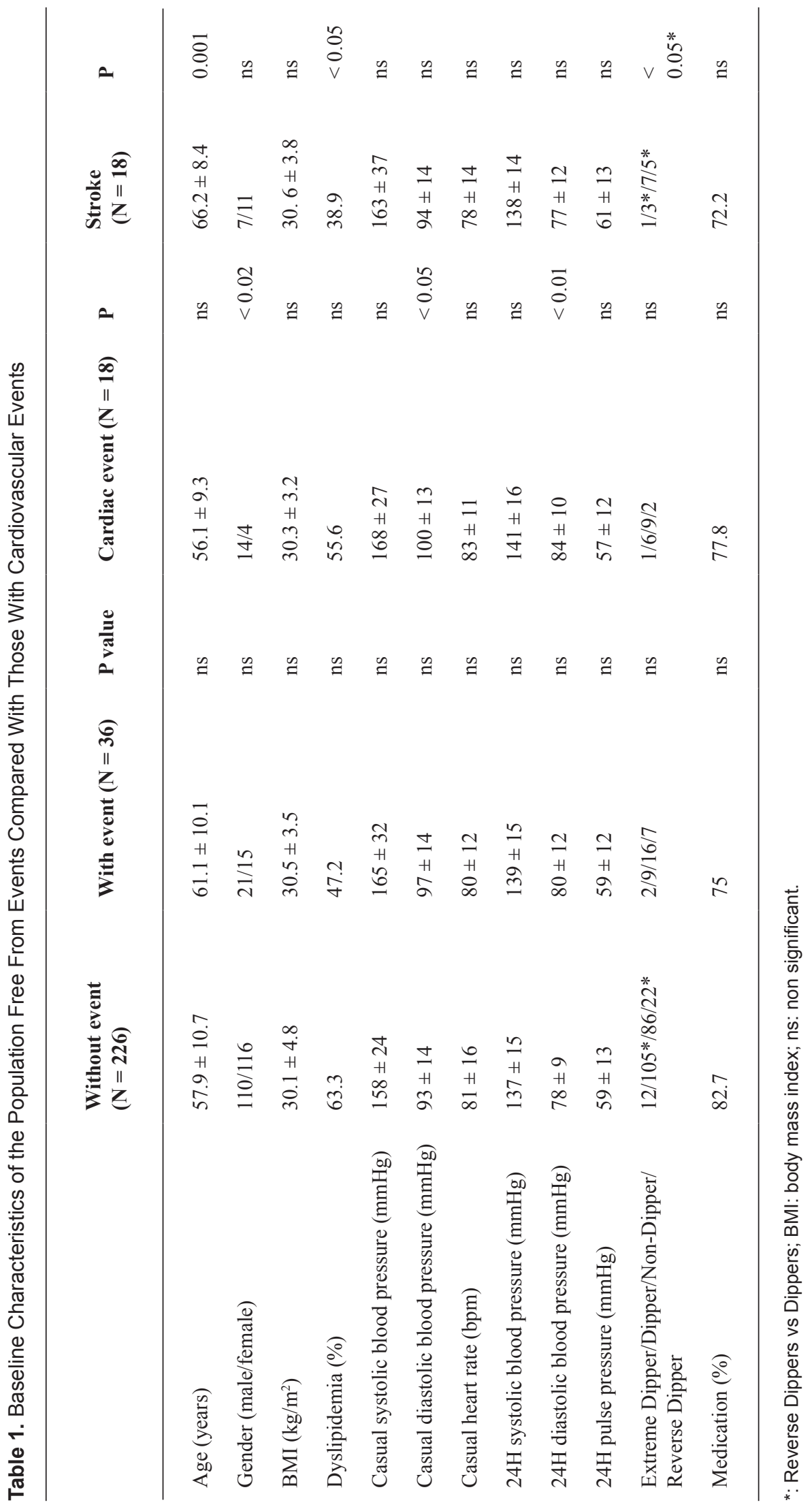


Table 2. Characteristics of the Nocturnal Fall Patterns, Showing Significant Differences Only Between the Reverse Dipper and Dipper Patterns, in Relation to Age *

\begin{tabular}{|c|c|c|c|c|c|}
\hline & ED & D & ND & RD & P value \\
\hline Gender (male\%) & 50 & 54.4 & 49 & 41.4 & ns \\
\hline Age (years) & 61.3 & 56.3 & 59.1 & 61.3 & $<0.05^{*}$ \\
\hline Dyslipidemia (\%) & 85.7 & 63.2 & 60.8 & 48.3 & ns \\
\hline Medication (\%) & 78.6 & 83.3 & 81.4 & 75.9 & ns \\
\hline BMI $\left(\mathrm{kg} / \mathrm{m}^{2}\right)$ & 32.0 & 30.9 & 29.4 & 28.6 & ns \\
\hline Casual SBP (mmHg) & 167 & 159 & 159 & 150 & ns \\
\hline Casual DBP (mmHg) & 99 & 94 & 93 & 88 & ns \\
\hline 24H SBP (mmHg) & 134 & 134 & 140 & 139 & $\mathrm{~ns}$ \\
\hline 24H DBP $(\mathrm{mmH}$ & 75 & 78 & 79 & 78 & ns \\
\hline 24H PP (mmHg) & 59 & 57 & 60 & 61 & ns \\
\hline
\end{tabular}

ED: extreme dipper; D: dipper; ND: non-dipper; RD: reverse dipper; BMI: body mass index; SBP: systolic BP; DBP: diastolic BP; PP: pulse pressure.

cardiovascular events.

\section{Methods}

\section{Subjects}

It was a retrospective observational study including 262 type 2 diabetic patients that underwent a AMBP recording between June 1991 and 2010, under stabilized medication, who were followed for $4.7 \pm 3.5$ years thereafter. Subjects were referred to confirm the presence of hypertension, to evaluate its severity, or to follow up on response to therapy; they had to be older than 18 years, and could not have a history of any previous cardiovascular event. The weight of each AMBP was considered until the occurrence of a first cardiovascular event.

\section{Ambulatory monitoring}

The AMBP was measured by an oscillometric technique (Spacelabs 90207 - SpaceLabs Inc, Redmond, Washington, USA). The monitor was mounted on the nondominant arm between 08.00 and $10.00 \mathrm{am}$, and removed $24 \mathrm{~h}$ later; the intervals between readings were 20 minutes during the day and 30 minutes at night. Subjects were encouraged to pursue their routine activities. Sleep and awake hours were recorded as reported in the subject's diary. If more than $85 \%$ of the readings were missing, the AMBP was excluded. The BP nocturnal fall patterns were considered as defined elsewhere [15].

\section{Cardiovascular events}

The end-point was a combination of cardiac and cerebrovascular events. Any subject was considered as having a cardiovascular event if one of the following criteria was present in the computer registry of our hospital: myocardial infarction, fatal or non-fatal, coronary artery bypass grafting or percutaneous transluminal coronary angioplasty or acute stroke, fatal or non-fatal. All patients were contacted by telephone to ascertain the absence of other events. The definition of dyslipidemia was made based on the previous diagnostic, medication with statins or elevated LDL cholesterol values at the time of the AMBP.

\section{Statistical analysis}

Continuous variables were correlated using Student's T test. When analysis of variance (ANOVA) indicated significant differences between groups, pair wise comparisons were assessed with significance levels appropriately modified us- 
Global Cardiovascular Events

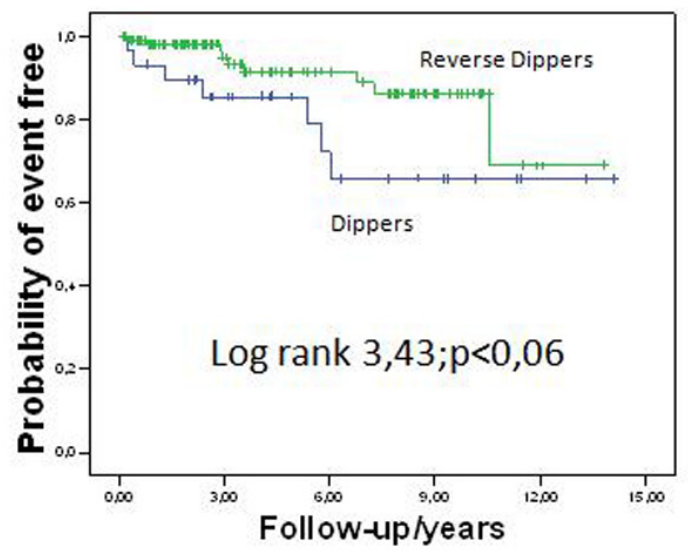

Stroke

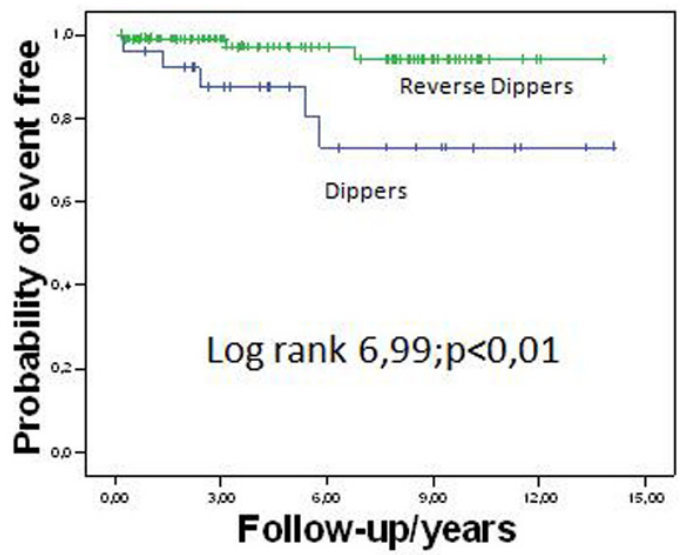

Figure 1. Kaplan-Meier curves showing survival-free from global cardiovascular events and stroke, according to Dipper or Reverse Dipper patterns of nocturnal fall.

ing the method of Games-Howell (two-sided). For noncontinuous variables the $\chi^{2}$ test was used. Multiple regression analysis was performed by Cox regression models, using as confounding variables age, gender, smoking, presence of dyslipidemia, anti-hypertensive medication and casual systolic BP. Survival and event-free survival curves were estimated using the Kaplan-Meier product-limit method and compared by the log-rank test. A two-tailed P value of less than 0.05 was considered significant. Results are expressed as mean $\pm \mathrm{SD}$.

\section{Results}

Baseline data are shown in Table 1.

During the follow-up there were 36 cardiovascular events, 11 acute coronary events, one of them fatal, 7 coronary revascularizations, and 18 strokes (predominantly ischemic, but not all characterized), five of which fatal. The global mortality rate was $2.3 \%$.

Groups with and without events did not differ significantly. In the subgroup analysis, coronary events were more frequent both in men and in patients with higher casual and 24H diastolic BP; patients within the stroke subgroup were older and had a lower prevalence of dyslipidemia and a higher prevalence of the reverse dipper pattern as compared to the dipper one.

The demographic and clinical characteristics of the nocturnal fall patterns are shown in Table 2.

As shown, the non-dipping patterns were present in about half the patients, accounting the reverse dippers for $11 \%$ of the total. Also, besides reverse dipper patients were older than the dipper ones, no other significant differences were found between groups.

As shown in Figure 1, the unadjusted event-free survival from stroke, given by the Kaplan-Meier curves, was higher

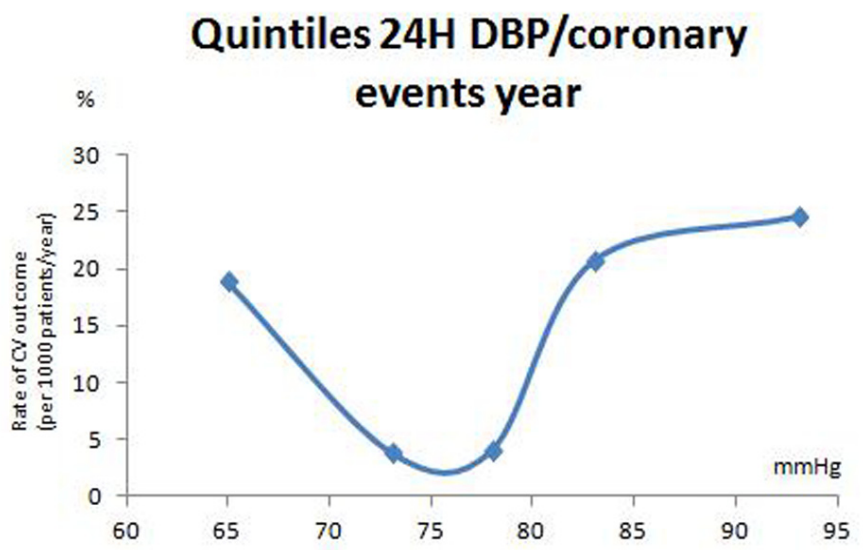

Figure 2. Rate of occurrence of coronary events according to the quintiles of $24 \mathrm{H}$ diastolic blood pressure. 


\section{Global Cardiovascular Events}

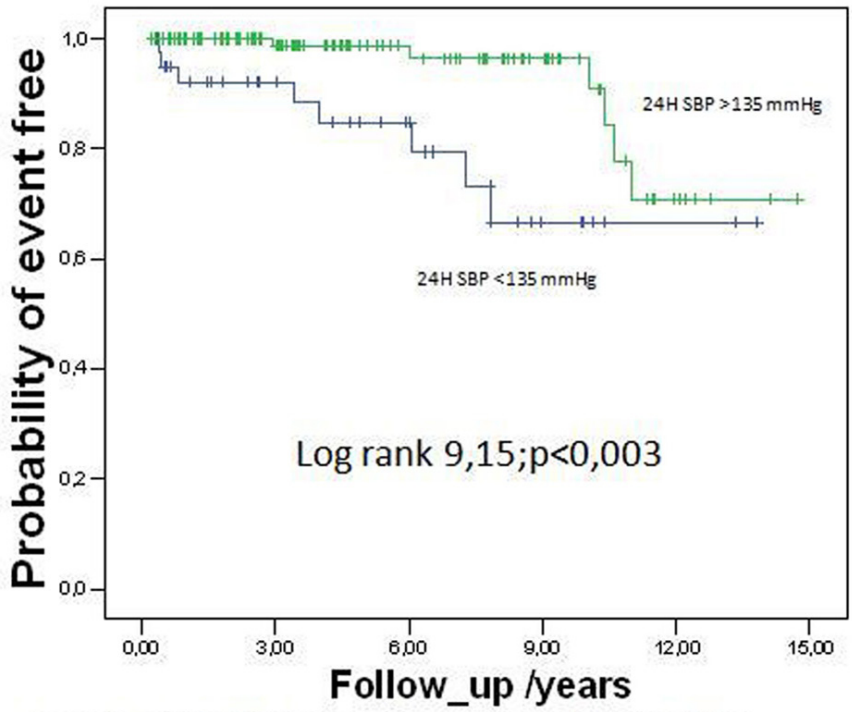

Figure 3. Kaplan-Meier curves showing survival-free from global cardiovascular events in the subgroup with $24 \mathrm{H}$ diastolic BP $\geq 75 \mathrm{mmHg}$, according to different levels of $24 \mathrm{H}$ systolic BP.

in the dipper patients as compared to the reverse dipper ones (log rank 6.99; $\mathrm{P}<0.01$ ), with a tendency to higher values also in the global event-free survival (log rank 3.43 ; $\mathrm{P}<$ 0.06 ).

In the multivariate Cox regression analysis, the only independent predictors of total events were age, for global events and stroke, hazard ratios (HR) respectively 1.04 (95\%CI 1.00 - 1.07) $\mathrm{P}=0.05$ and 1.09 (95\%CI $1.03-1.14)$ $\mathrm{P}<0.01$, and male gender for coronary events, HR 4.01 $(95 \%$ CI $1.26-12.73) \mathrm{P}<0.02$. Casual systolic BP was also an independent predictor of coronary events, but lost its significance when casual diastolic BP and the AMBP parameters were considered.

The subdivision of the 24-hour systolic and diastolic BP in their quintiles of distribution, rendered a $J$ shaped curve to the diastolic BP distribution, for global and coronary events (Fig. 2), with a nadir at about $75 \mathrm{mmHg}$.

As shown in Figure 3, 24H systolic BP (above and below $135 \mathrm{mmHg}$ ) significantly and independently predicted cardiovascular events in the subpopulation with $24 \mathrm{H}$ diastolic $\mathrm{BP}>75 \mathrm{mmHg}$ (HR 0.30; 95\%CI $0.09-0.57$; $>0.002)$, showing decreased event-free survival in the Kaplan-Meier curves (log rank 9.15; $\mathrm{P}<0.003)$.

Figure 4 shows the rate of occurrence of total cardiovascular events according to the quintiles of $24 \mathrm{H}$ diastolic blood pressure after excluding patients with isolated systolic hypertension, namely SBP $\geq 140$ and $\mathrm{DBP}<90 \mathrm{mmHg}$ (namely $25.6 \%$ of the population). As shown in that population we still encountered a $\mathrm{J}$ shaped curve in the distribution of the $24 \mathrm{H}$ diastolic BP, for global and coronary events, with a similar nadir as for the overall diabetic population shown in Figure 2.

\section{Discussion}

In our population of type 2 diabetics without previous cardiovascular events we found a clear $\mathbf{J}$ shaped curve to the distribution of the 24-hour diastolic blood pressure for global cardiovascular events. Cruickshank et al [13] had previously showed that fatal myocardial infarction increased in patients with casual diastolic BP lower than $85 \mathrm{mmHg}$, particularly if they had coronary ischemia, mainly through a diminished coronary perfusion reserve [16]. Although our population could be considered as a high-risk population, similar data have been showed in patients without proved ischemia [14], but few studies have utilized the AMBP parameters in this particular subset.

Our results show some particularities. Firstly, we found the 24-h DBP J shaped curve occurred not only for coronary events, but also for global events, with the natural contribution of strokes, despite acknowledgment that the cerebral circulation is not so dependent on the diastolic phase of flow as compared with coronary circulation.

Secondly, the value of the nadir rendering a lower prevalence of events was $75 \mathrm{mmHg}$. This value, which is lower than that reported previously [17], was achieved in a nondespicable portion of the diabetic population. That could be related to the measurement by AMBP instead of casual $\mathrm{BP}$ for which the normalcy values of the former are usually 


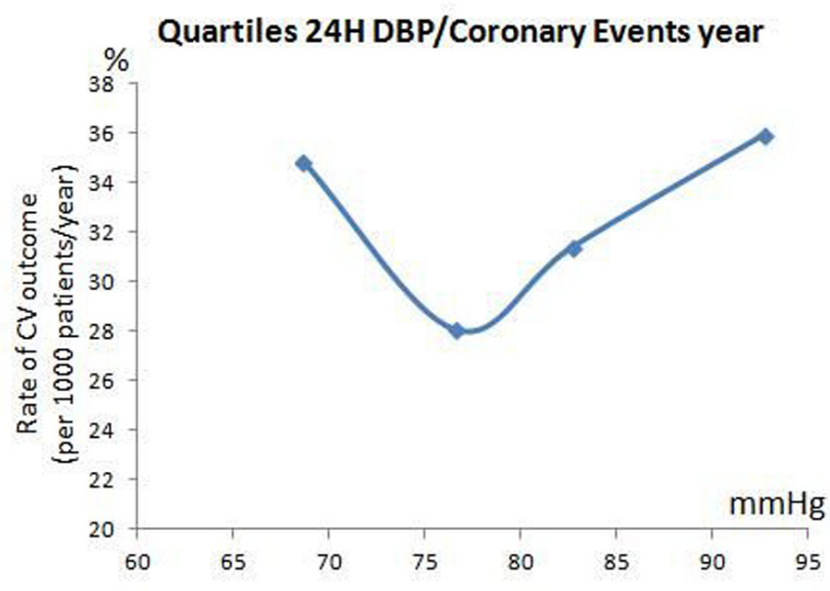

Figure 4 - Rate of occurrence of total cardiovascular events according

to the quintiles of $24 \mathrm{H}$ diastolic blood pressure (excluding isolated

systolic hypertensives).

Figure 4. Rate of occurrence of total cardiovascular events according to the quintiles of $24 \mathrm{H}$ diastolic blood pressure (excluding isolated systolic hypertensives).

lower that of the latter. Another explanation could be related to a higher prevalence of larger pulse pressures in diabetic patients, parameter with well known prognostic implications [18] or to a higher prevalence of isolated systolic hypertension with increasing age, for example, a demographic risk factor that independently predicted events in our population. However, when isolated systolic hypertension was excluded from the analysis (Fig. 4), we still found a J shaped curve, which, therefore, diminished its role in the phenomenon.

Moreover, and in relation to that point, we also found that high 24 hours systolic BP values, which independently predicted events in the multivariate analysis and decreased the event-free survival in the Kaplan-Meier curves, only did so when 24 hours diastolic BP values were normal or high. In 2004, Kannel et al [19] reanalyzed the Framingham data, and claimed the $\mathrm{J}$ curve phenomenon for diastolic BP $(<80$ $\mathrm{mmHg}$ ) only occurred in the presence of elevated systolic BP (>140 $\mathrm{mmHg}$ ), after adjustment for age and other risk factors, emphasizing the role of pulse pressure. In our population that was not the case, since low diastolic BP values had an impact on its own, even in a somewhat aged population, which so far [20] wasn't considered a risk factor by itself.

One of the major prognostic contributions of AMBP is related to the information about $\mathrm{BP}$ circadian rhythm, that may be particularly important in the diabetic population [2, 21]. There is a higher prevalence of the non-dipper pattern in diabetics when compared to the general population [22]. That appears not to be just dependent on the quality of sleep, as showed by Perk et al [23], who found a blunted decline of BP during the afternoon siesta, namely a short period of sleep that is less dependent on sleep quality. Otherwise, it was suggested [23] the phenomenon to be a problem of the sleep itself as a facet of autonomic dysfunction. In our population, non-dipping patterns (non-dippers and reverse dippers) were very prevalent, accounting for half of the population. The reverse dipper pattern, that was present in $11 \%$ of patients, was a factor of disadvantage in the stroke event-free survival when compared with the dipper patients, thereby showing a tendency as a drawback for global events. In general this is in agreement to our previous studies [24]. Two recent reviews $[25,26]$ also point out the prognostic disadvantages of the reverse dipper pattern while being an independent predictor of macrovascular as well as microvascular events. The question remains whether such a reverse dipping pattern is the cause of the events or just a marker of the progression of the disease. In fact, autonomic dysfunction [27, 28] or the necessity of greater sodium nocturnal excretion [29], are among the mechanisms that have been implicated in the blunting or reversion of nighttime BP fall.

Although other patterns of BP circadian rhythm, such as the extreme nocturnal blood pressure dippers, have also been related with a higher risk of ischemic stroke [30], in our study the small number of extreme dippers unable us to examine such a relationship. In any case, the importance of the BP circadian rhythm in the global evaluation of the diabetics is becoming overwhelming, independently of the remaining parameters/indexes of the AMBP.

The study has some limitations particularly related with the small population sample and the low incidence of events that have occurred in our population. Also, since only one 
ABPM recording was done, we could not account for any change in habits or in therapy that may have been done during the follow-up period. Nevertheless, these are limitations shared in common to the other few studies that have examined the prognostic values of AMBP in diabetic patients.

In conclusion, in diabetic patients without previous cardiovascular events we have found a clear J shaped curve for the distribution of 24 hour diastolic BP in relation to global and coronary events, that was independent of $24 \mathrm{H}$ systolic $\mathrm{BP}$ and pulse pressure values, and of isolated systolic hypertension. Our data suggest that in diabetic patients low $24 \mathrm{H}$ diastolic BP values are associated with increased cardiovascular risk and must be carefully followed. Furthermore, the nocturnal reverse dipper pattern that is highly prevalent in diabetics also has negative prognostic implications for stroke and also possibly for other cardiovascular events, and should be monitored.

\section{References}

1. Wild S, Roglic G, Green A, Sicree R, King H. Global prevalence of diabetes: estimates for the year 2000 and projections for 2030. Diabetes Care. 2004;27(5):10471053.

2. Astrup AS, Nielsen FS, Rossing P, Ali S, Kastrup J, Smidt UM, Parving HH. Predictors of mortality in patients with type 2 diabetes with or without diabetic nephropathy: a follow-up study. J Hypertens. 2007;25(12):2479-2485.

3. Laakso M. Hyperglycemia and cardiovascular disease in type 2 diabetes. Diabetes. 1999;48(5):937-942.

4. Volpe M, Tocci G. 2007 ESH/ESC Guidelines for the management of hypertension, from theory to practice: global cardiovascular risk concept. J Hypertens Suppl. 2009;27(3):S3-11.

5. Tight blood pressure control and risk of macrovascular and microvascular complications in type 2 diabetes: UKPDS 38. UK Prospective Diabetes Study Group. BMJ. 1998;317(7160):703-713.

6. Hansen TW, Jeppesen J, Rasmussen S, Ibsen H, Torp-Pedersen C. Ambulatory blood pressure and mortality: a population-based study. Hypertension. 2005;45(4):499-504.

7. Eguchi K, Pickering TG, Hoshide S, Ishikawa J, Ishikawa S, Schwartz JE, Shimada K, et al. Ambulatory blood pressure is a better marker than clinic blood pressure in predicting cardiovascular events in patients with/without type 2 diabetes. Am J Hypertens. 2008;21(4):443450.

8. Knudsen ST, Poulsen PL, Hansen KW, Ebbehoj E, Bek T, Mogensen CE. Pulse pressure and diurnal blood pressure variation: association with micro- and macrovascular complications in type 2 diabetes. Am J Hypertens. 2002;15(3):244-250.

9. Sega R, Corrao G, Bombelli M, Beltrame L, Facchetti
R, Grassi G, Ferrario M, et al. Blood pressure variability and organ damage in a general population: results from the PAMELA study (Pressioni Arteriose Monitorate E Loro Associazioni). Hypertension. 2002;39(2 Pt 2):710714.

10. Mesquita-Bastos J, Bertoquini S, Polonia J. Cardiovascular prognostic value of ambulatory blood pressure monitoring in a Portuguese hypertensive population followed up for 8.2 years. Blood Press Monit. 2010;15(5):240-246.

11. Knudsen ST, Laugesen E, Hansen KW, Bek T, Mogensen CE, Poulsen PL. Ambulatory pulse pressure, decreased nocturnal blood pressure reduction and progression of nephropathy in type 2 diabetic patients. Diabetologia. 2009;52(4):698-704.

12. Anderson TW. Re-examination of some of the Framingham blood-pressure data. Lancet. 1978;2(8100):11391141 .

13. Cruickshank JM, Thorp JM, Zacharias FJ. Benefits and potential harm of lowering high blood pressure. Lancet. 1987;1(8533):581-584.

14. Waller PC, Isles CG, Lever AF, Murray GD, McInnes GT. Does therapeutic reduction of diastolic blood pressure cause death from coronary heart disease? J Hum Hypertens. 1988;2(1):7-10.

15. Kario K, Matsuo T, Kobayashi H, Imiya M, Matsuo M, Shimada K. Nocturnal fall of blood pressure and silent cerebrovascular damage in elderly hypertensive patients. Advanced silent cerebrovascular damage in extreme dippers. Hypertension. 1996;27(1):130-135.

16. Cruickshank JM. Coronary flow reserve and the J curve relation between diastolic blood pressure and myocardial infarction. BMJ. 1988;297(6658):1227-1230.

17. Farnett L, Mulrow CD, Linn WD, Lucey CR, Tuley MR. The J-curve phenomenon and the treatment of hypertension. Is there a point beyond which pressure reduction is dangerous? JAMA. 1991;265(4):489-495.

18. Nakano S, Ito T, Furuya K, Tsuda S, Konishi K, Nishizawa M, Nakagawa A, et al. Ambulatory blood pressure level rather than dipper/nondipper status predicts vascular events in type 2 diabetic subjects. Hypertens Res. 2004;27(9):647-656.

19. Kannel WB, Wilson PW, Nam BH, D’Agostino RB, Li J. A likely explanation for the J-curve of blood pressure cardiovascular risk. Am J Cardiol. 2004;94(3):380-384.

20. Franklin SS, Larson MG, Khan SA, Wong ND, Leip EP, Kannel WB, Levy D. Does the relation of blood pressure to coronary heart disease risk change with aging? The Framingham Heart Study. Circulation. 2001;103(9):1245-1249.

21. Bursztyn M, Ben-Dov IZ. Diabetes mellitus and 24-hour ambulatory blood pressure monitoring: broadening horizons of risk assessment. Hypertension. 2009;53(2):110111. 
22. Palmas W, Pickering TG, Teresi J, Schwartz JE, Moran A, Weinstock RS, Shea S. Ambulatory blood pressure monitoring and all-cause mortality in elderly people with diabetes mellitus. Hypertension. 2009;53(2):120127.

23. Perk G, Mekler J, Ben Ishay D, Bursztyn M. Non-dipping in diabetic patients: insights from the siesta. J Hum Hypertens. 2002;16(6):435-438.

24. Bastos JM, Bertoquini S, Polonia J. Prognostic value of subdivisions of nighttime blood pressure fall in hypertensives followed up for 8.2 years. Does nondipping classification need to be redefined? J Clin Hypertens (Greenwich). 2010;12(7):508-515.

25. Pierdomenico SD, Cuccurullo F. Ambulatory blood pressure monitoring in type 2 diabetes and metabolic syndrome: a review. Blood Press Monit. 2010;15(1):1-7.

26. Eguchi K. Ambulatory blood pressure monitoring in diabetes and obesity-a review. Int J Hypertens. 2011;2011(954757.

27. Polonia J, Amaral C, Bertoquini S, Martins L. Attenuation of heart rate recovery after exercise in hypertensive patients with blunting of the nighttime blood pressure fall. Int J Cardiol. 2006;106(2):238-243.

28. Dodt C, Breckling U, Derad I, Fehm HL, Born J. Plasma epinephrine and norepinephrine concentrations of healthy humans associated with nighttime sleep and morning arousal. Hypertension. 1997;30(1 Pt 1):71-76.

29. Pickering TG, Kario K. Nocturnal non-dipping: what does it augur? Curr Opin Nephrol Hypertens. 2001;10(5):611-616.

30. Kario K, Pickering TG, Matsuo T, Hoshide S, Schwartz JE, Shimada K. Stroke prognosis and abnormal nocturnal blood pressure falls in older hypertensives. Hypertension. 2001;38(4):852-857. 